ARTICLE

DOI: $10.1038 / \mathrm{s} 41467-018-07522-1$

OPEN

\title{
A fast quantum interface between different spin qubit encodings
}

\author{
A. Noiri (1) 1, T. Nakajima (1) 1, J. Yoneda (1) 1, M.R. Delbecq (1) 1,2, P. Stano1, T. Otsuka (1) 1,3,4, K. Takeda (1) 1, \\ S. Amaha (1) ${ }^{1}$, G. Allison ${ }^{1}$, K. Kawasaki ${ }^{5}$, Y. Kojima ${ }^{5}$, A. Ludwig (1) ${ }^{6}$, A.D. Wieck (1) ${ }^{6}$, D. Loss (1) ${ }^{1,7} \&$ S. Tarucha ${ }^{1,5}$
}

Single-spin qubits in semiconductor quantum dots hold promise for universal quantum computation with demonstrations of a high single-qubit gate fidelity above $99.9 \%$ and twoqubit gates in conjunction with a long coherence time. However, initialization and readout of a qubit is orders of magnitude slower than control, which is detrimental for implementing measurement-based protocols such as error-correcting codes. In contrast, a singlet-triplet qubit, encoded in a two-spin subspace, has the virtue of fast readout with high fidelity. Here, we present a hybrid system which benefits from the different advantages of these two distinct spin-qubit implementations. A quantum interface between the two codes is realized by electrically tunable inter-qubit exchange coupling. We demonstrate a controlled-phase gate that acts within $5.5 \mathrm{~ns}$, much faster than the measured dephasing time of $211 \mathrm{~ns}$. The presented hybrid architecture will be useful to settle remaining key problems with building scalable spin-based quantum computers.

\footnotetext{
${ }^{1}$ RIKEN, Center for Emergent Matter Science (CEMS), Wako-shi, Saitama 351-0198, Japan. ${ }^{2}$ Laboratoire Pierre Aigrain, Ecole Normale Supérieure-PSL Research University, CNRS, Université Pierre et Marie Curie-Sorbonne Universités, Université Paris Diderot-Sorbonne Paris Cité, 24 rue Lhomond, 75231 Paris Cedex 05, France. ${ }^{3}$ Research Institute of Electrical Communication, Tohoku University, 2-1-1 Katahira, Aoba-ku, Sendai 980-8577, Japan. ${ }^{4}$ JST, PRESTO, 4-18 Honcho, Kawaguchi, Saitama 332-0012, Japan. ${ }^{5}$ Department of Applied Physics, University of Tokyo, 7-3-1 Hongo, Bunkyo-ku, Tokyo 113-8656, Japan. ${ }^{6}$ Lehrstuhl für Angewandte Festkörperphysik, Ruhr-Universität Bochum, D-44780 Bochum, Germany. ${ }^{7}$ Department of Physics, University of Basel, Klingelbergstrasse 82, 4056 Basel, Switzerland. These authors contributed equally: A. Noiri, T. Nakajima. Correspondence and requests for materials should be addressed to A.N. (email: akito.noiri@riken.jp) or to S.T. (email: tarucha@ap.t.u-tokyo.ac.jp)
} 
nitialization, single-qubit and two-qubit gate operations, and measurements are fundamental elements for universal quantum computation ${ }^{1}$. Generally, they should all be fast and with high fidelity to reach the fault-tolerance thresholds ${ }^{2}$. So far, various encodings of spin qubits into one to three-spin subspaces have been developed in semiconductor quantum dots ${ }^{3-15}$. In particular, recent experiments demonstrated all of these elements including two-qubit logic gates for single-spin qubits proposed by Loss and DiVincenzo (LD qubits) and singlet-triplet (ST) qubits ${ }^{6-}$ 8,14 . These qubits have different advantages depending on the gate operations, and combinations thereof can increase the performance of spin-based quantum computing. In LD qubits, the twoqubit gate is fast ${ }^{6,7}$ as it relies on the exchange interaction between neighboring spins. In contrast, the two-qubit gate in ST qubits is much slower as it is mediated by a weak dipole coupling ${ }^{14}$. Concerning initialization and readout, however, the situation is the opposite: it is slow for LD qubits, relying on spinselective tunneling to a lead ${ }^{16,17}$, while it is orders of magnitude faster in ST qubits relying on Pauli spin blockade ${ }^{12,13}$. Therefore, a fast and reliable interface between LD and ST qubits would allow for merging the advantages of both realizations.

Here we present such an interface implementing a controlledphase (CPHASE) gate between a LD qubit and a ST qubit in a quantum dot array ${ }^{18,19}$. The gate is based on the nearest neighbor exchange coupling and is performed in $5.5 \mathrm{~ns}$. Even though we do not pursue benchmarking protocols here, the gate time being much shorter than the corresponding dephasing time (211 ns) indicates that the fidelity of this type of gates can be very high. Our results demonstrate that controlled coherent coupling of different types of gated spin qubits is feasible, and one can proceed to combining their advantages. Overall, our work pushes further the demonstrated scalability of spin qubits in quantum dot arrays.

\section{Results}

A LD qubit and a ST qubit formed in a triple quantum dot (TQD). A hybrid system comprising a LD qubit and a ST qubit is implemented in a linearly-coupled gate-defined TQD shown in Fig. 1a. The $\mathrm{LD}$ qubit $\left(\mathrm{Q}_{\mathrm{LD}}\right)$ is formed in the left dot while the ST qubit $\left(\mathrm{Q}_{\mathrm{ST}}\right)$ is hosted in the other two dots. We place a micromagnet near the TQD to coherently and resonantly control $\mathrm{Q}_{\mathrm{LD}}$ via electric dipole spin resonance (EDSR) ${ }^{20-23,26}$. At the same time it makes the Zeeman energy difference between the center and right dots, $\Delta E_{\mathrm{Z}}^{\mathrm{ST}}$, much larger than their exchange coupling $J^{S T}$, such that the eigenstates of $\mathrm{Q}_{\mathrm{ST}}$ become $|\uparrow \downarrow\rangle$ and $|\downarrow \uparrow\rangle$ rather than singlet $|S\rangle$ and triplet $|\mathrm{T}\rangle$. We apply an external in-plane magnetic field $B_{\text {ext }}=3.166 \mathrm{~T}$ to split the $\mathrm{Q}_{\mathrm{LD}}$ states by the Zeeman energy $E_{\mathrm{Z}}$ as well as to separate polarized triplet states $|\uparrow \uparrow\rangle$ and $|\downarrow \downarrow\rangle$ from the $\mathrm{Q}_{\mathrm{ST}}$ computational states. The experiment is conducted in a dilution refrigerator with an electron temperature of approximately $120 \mathrm{mK}$. The qubits are manipulated in the $\left(\mathrm{N}_{\mathrm{L}}\right.$, $\left.\mathrm{N}_{\mathrm{C}}, \mathrm{N}_{\mathrm{R}}\right)=(1,1,1)$ charge state while the $(1,0,1)$ and $(1,0,2)$ charge states are also used for initialization and readout (see Fig. 1b). Here, $\mathrm{N}_{\mathrm{L}(\mathrm{C}, \mathrm{R})}$ denotes the number of electrons inside the left (center, right) dot.

We first independently measure the coherent time evolution of each qubit to calibrate the initialization, control, and readout. We quench the inter-qubit exchange coupling by largely detuning the energies of the $(1,1,1)$ and $(2,0,1)$ charge states. For $\mathrm{Q}_{\mathrm{LD}}$, we observe Rabi oscillations ${ }^{4}$ with a frequency $f_{\text {Rabi }}$ of up to $10 \mathrm{MHz}$ (Fig. 1d) as a function of the microwave (MW) burst time $t_{\mathrm{MW}}$, using the pulse sequence in Fig. 1e. For $\mathrm{Q}_{\mathrm{ST}}$, we observe the precession between $|\mathrm{S}\rangle$ and $|\mathrm{T}\rangle$ (ST precession) (Fig. 1f) as a function of the evolution time $t_{\mathrm{e}}$, using the pulse sequence in Fig. $1 \mathrm{~g}$ (see Supplementary Note 2 for full control of $\mathrm{Q}_{\mathrm{ST}}$ ). We use a metastable state to measure $\mathrm{Q}_{\mathrm{ST}}$ with high fidelity ${ }^{13}$ (projecting to $|\mathrm{S}\rangle$ or $|\mathrm{T}\rangle)$ in the presence of large $\Delta E_{\mathrm{Z}}^{\mathrm{ST}}$ with which the lifetime of $|\mathrm{T}\rangle$ is $\operatorname{short}^{27}$.

Calibration of the two-qubit coupling. The two qubits are interfaced by exchange coupling $J^{\mathrm{QQ}}$ between the left and center dots as illustrated in Fig. 1c. We operate the two-qubit system under the conditions of $E_{\mathrm{Z}} \gg \Delta E_{\mathrm{Z}}^{\mathrm{ST}}, \Delta E_{\mathrm{Z}}^{\mathrm{QQ}} \gg J^{\mathrm{QQ}} \gg J^{\mathrm{ST}}$ where $\Delta E_{\mathrm{Z}}^{\mathrm{QQ}}$ is the Zeeman energy difference between the left and center dots. Then, the Hamiltonian of the system is

$$
\mathcal{H}=-E_{\mathrm{Z}} \hat{\sigma}_{z}^{\mathrm{LD}} / 2-\Delta E_{\mathrm{Z}}^{\mathrm{ST}} \hat{\sigma}_{z}^{\mathrm{ST}} / 2+J^{\mathrm{QQ}}\left(\hat{\sigma}_{z}^{\mathrm{LD}} \hat{\sigma}_{z}^{\mathrm{ST}}-1\right) / 4
$$

where $\hat{\sigma}_{z}^{\mathrm{LD}}$ and $\hat{\sigma}_{z}^{\mathrm{ST}}$ are the Pauli z-operators of $\mathrm{Q}_{\mathrm{LD}}$ and $\mathrm{Q}_{\mathrm{ST}}$, respectively ${ }^{18}$ (Supplementary Note 3 ). The last term in Eq. (1) reflects the effect of the inter-qubit coupling $J^{\mathrm{QQ}}$ : for states in which the spins in the left and center dots are antiparallel, the energy decreases by $J^{Q Q} / 2$ (see Fig. 2a). In the present work, we choose to operate $\mathrm{Q}_{\mathrm{LD}}$ as a control qubit and $\mathrm{Q}_{\mathrm{ST}}$ as a target, although these are exchangeable. With this interpretation, the ST precession frequency $f^{S T}$ depends on the state of $\mathrm{Q}_{\mathrm{LD}}$ $f_{\sigma_{z}^{\mathrm{LD}}}^{\mathrm{ST}}=\left(\Delta E_{\mathrm{Z}}^{\mathrm{ST}}-\sigma_{z}^{\mathrm{LD}} J^{\mathrm{QQ}} / 2\right) / h$. Here $\sigma_{z}^{\mathrm{LD}}$ represents $|\uparrow\rangle$ or $|\downarrow\rangle$ and +1 or -1 interchangeably. This means that while $J \mathrm{QQ}$ is turned on for the interaction time $t_{\text {int }}, \mathrm{Q}_{\mathrm{ST}}$ accumulates the controlled-phase $\phi_{\mathrm{C}}=2 \pi J^{\mathrm{QQ}} t_{\text {int }} / h$, which provides the CPHASE gate (up to singlequbit phase gates; see Supplementary Note 7) in $t_{\text {int }}=h / 2 J$ QQ. An important feature of this two-qubit gate is that it is intrinsically fast, scaling with $J^{\mathrm{QQ}} / h$ which can be tuned up to $\sim 100 \mathrm{MHz}$, and is limited only by the requirement $J^{\mathrm{QQ}} / h \ll \Delta E_{\mathrm{Z}}^{\mathrm{QQ}} / h \sim 500 \mathrm{MHz}$ in our device.

Before testing the two-qubit gate operations, we calibrate the inter-qubit coupling strength $\mathrm{JQQ}$, and its tunability by gate voltages. The inter-qubit coupling in pulse stage F (Fig. 2b) is controlled by the detuning energy between $(2,0,1)$ and $(1,1,1)$ charge states (one of the points denoted E in Fig. 1b). To prevent leakage from the $\mathrm{Q}_{\mathrm{ST}}$ computational states, we switch $\mathrm{JQ}^{\mathrm{QQ}}$ on and off adiabatically with respect to $\Delta E_{\mathrm{Z}}^{\mathrm{QQ}}$ by inserting voltage ramps to stage $\mathrm{F}$ with a total ramp time of $t_{\mathrm{ramp}}=24 \mathrm{~ns}$ (Fig. $\left.2 \mathrm{~b}\right)^{28}$. The coherent precession of $\mathrm{Q}_{\mathrm{ST}}$ is measured by repeating the pulse stages from $\mathrm{D}$ to $\mathrm{H}$ without initializing, controlling and measuring $\mathrm{Q}_{\mathrm{LD}}$, which makes $\mathrm{Q}_{\mathrm{LD}}$ a random mixture of $|\uparrow\rangle$ and $|\downarrow\rangle$. Figure $2 c$ shows the FFT spectra of the precession measured for various interaction points indicated in Fig. 1b. As we bring the interaction point closer to the boundary of $(1,1,1)$ and $(2,0,1), J \mathrm{QQ}$ becomes larger and we start to see splitting of the spectral peaks into two. The separation of the two peaks is given by $J \mathrm{QQ} / h$ which can be controlled by the gate voltage as shown in Fig. 2d.

We now demonstrate the controllability of the ST precession frequency by the input state of $\mathrm{Q}_{\mathrm{LD}}$, the essence of a CPHASE gate. We use the quantum circuit shown in Fig. $2 b$, which combines the pulse sequences for independent characterization of $\mathrm{Q}_{\mathrm{LD}}$ and $\mathrm{Q}_{\mathrm{ST}}$. Here we choose the interaction point such that $J^{\mathrm{QQ}} / h=90 \mathrm{MHz}$. By using either $|\uparrow\rangle$ or $|\downarrow\rangle$ as the $\mathrm{Q}_{\mathrm{LD}}$ initial state (the latter prepared by an $\operatorname{EDSR} \pi$ pulse), we observe the ST precessions as shown in Fig. 2e, f. The data fit well to Gaussian-decaying oscillations giving $f_{|\uparrow\rangle}^{\mathrm{ST}}=434 \pm 0.5 \mathrm{MHz}$ and $f_{|\downarrow\rangle}^{\mathrm{ST}}=524 \pm 0.4 \mathrm{MHz}$ [These are consistent with the values determined by Bayesian estimation discussed in Methods]. This demonstrates the control of the precession rate of $\mathrm{Q}_{\mathrm{ST}}$ by $J \mathrm{QQ} / h$ depending on the state of $\mathrm{Q}_{\mathrm{LD}}$.

Demonstration of a CPHASE gate. To characterize the controlled-phase accumulated during the pulse stage $\mathrm{F}$, we separate the phase of $\mathrm{Q}_{\mathrm{ST}}$ into controlled and single-qubit contributions as $\phi_{\sigma_{z}^{\mathrm{LD}}}=-\pi \sigma_{z}^{\mathrm{LD}} J^{\mathrm{QQ}}\left(t_{\mathrm{int}}+t_{0}\right) / h \quad$ and 


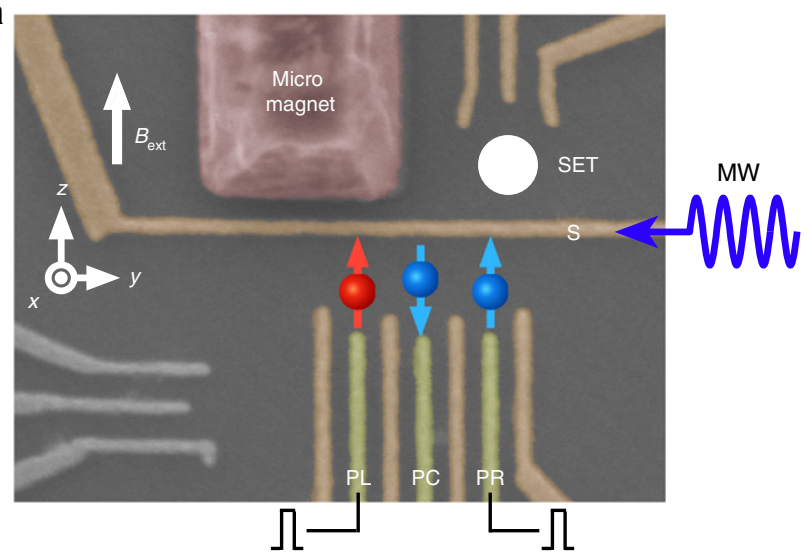

b



C

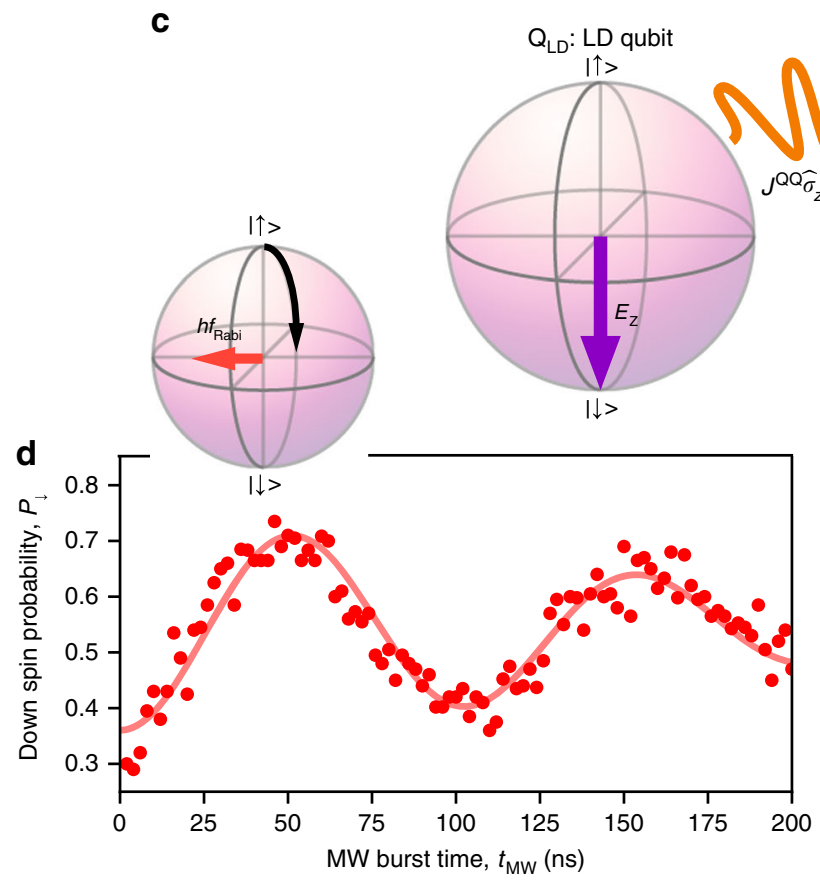

$\mathrm{Q}_{\mathrm{ST}}: \mathrm{ST}$ qubit
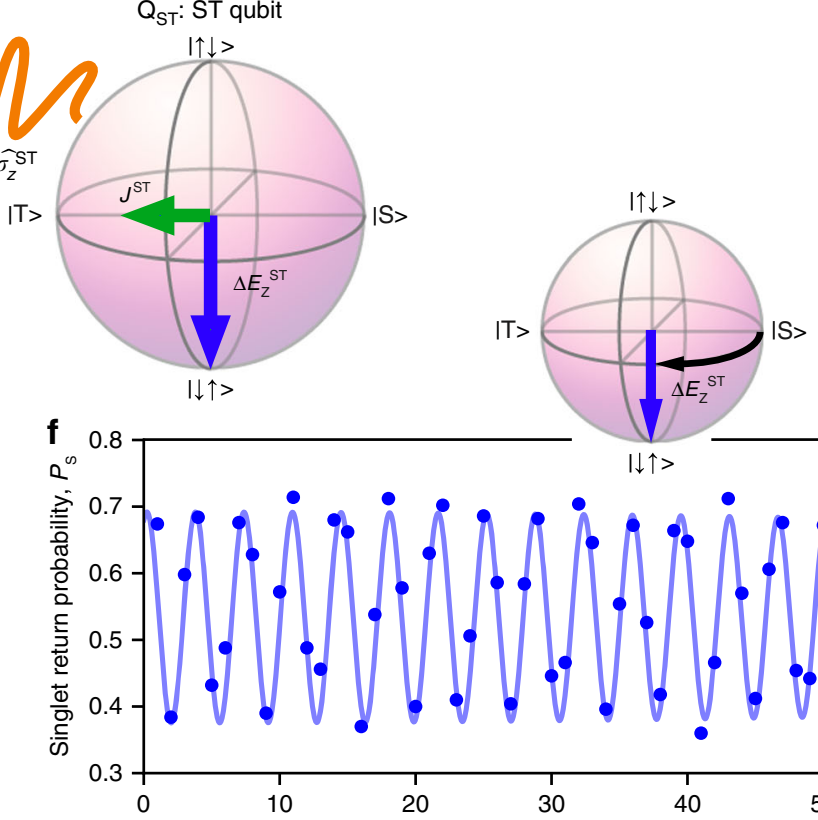

$\mid \downarrow \uparrow>$
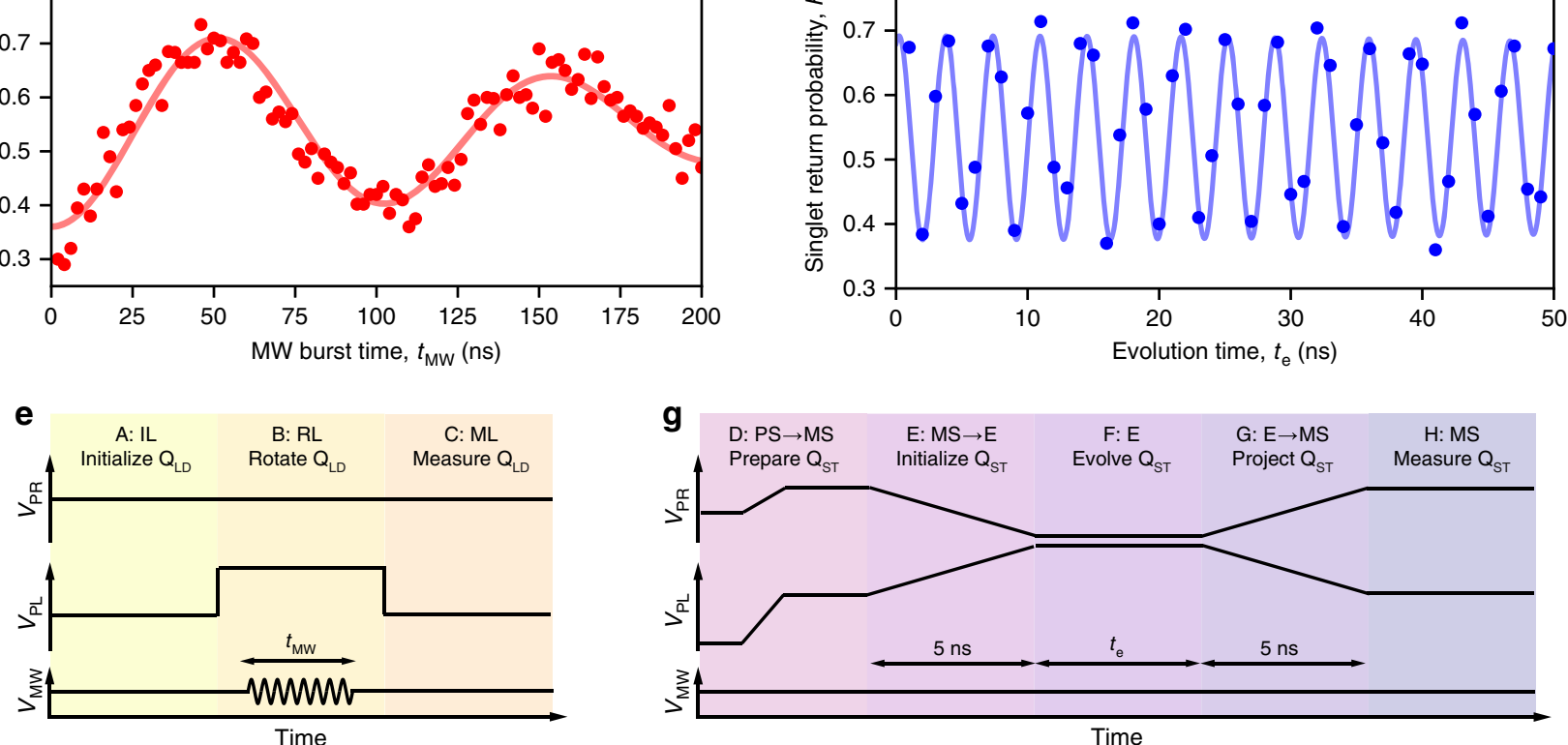

Fig. 1 Hybrid system of a LD qubit and a ST qubit realized in a TQD. a False color scanning electron microscope image of a device identical to the one used in this study. The TQD is defined in a two-dimensional electron gas at the GaAs/AIGaAs heterointerface $100 \mathrm{~nm}$ below the surface. The upper single electron transistor is used for radiofrequency-detected charge sensing 24,25 . A MW with a frequency of $17.26 \mathrm{GHz}$ is applied to the $\mathrm{S}$ gate to drive EDSR. b Stability diagram of the TQD obtained by differentiating the charge sensing signal $V_{\text {rf. }}$ c Hybrid system of a LD qubit and a ST qubit coupled by the exchange coupling JQQ. d Rabi oscillation of QLD (rotation around $x$-axis) driven by EDSR with $J Q Q \sim 0$ at point RL in Fig. 1b. The data is fitted to oscillations with a Gaussian decay of $T_{2}^{\text {Rabi }}=199 \mathrm{~ns}$. e Pulse sequence used to produce Fig. 1d showing gate voltages $V_{P L}$ and $V_{P R}$ applied to the PL and PR gates and a $\mathrm{MW}$ burst $V_{\mathrm{MW}}$. $\mathbf{f}$ Precession of $\mathrm{Q}_{\mathrm{ST}}$ (rotation around z-axis) with a frequency of $f^{\mathrm{ST}}=280 \mathrm{MHz}$ due to $\Delta E_{\mathrm{Z}}^{\mathrm{ST}}$, taken at point $E$ marked by the white circle in $(1,1,1)$ in Fig. 1b, where JQQ and JST $\sim 0$. The data follow the Gaussian decay with a decay time of 207 ns (see Supplementary Fig. 2a) induced by the nuclear field fluctuations ${ }^{29}$. g Pulse sequence used to produce Fig. If 
a

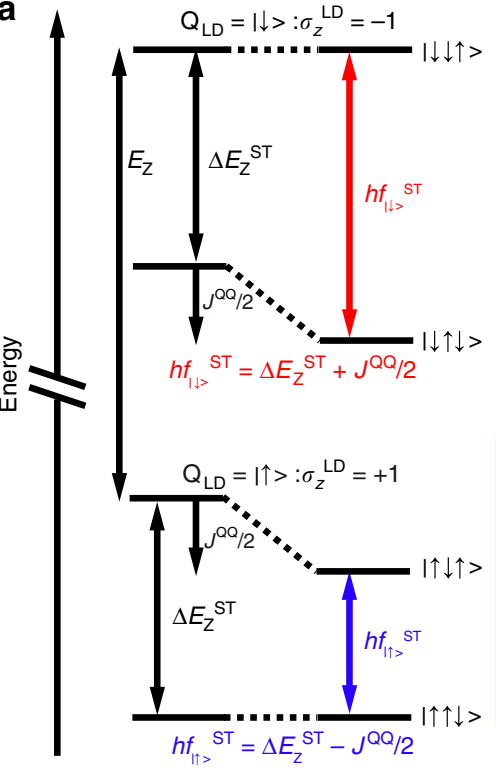

C



b

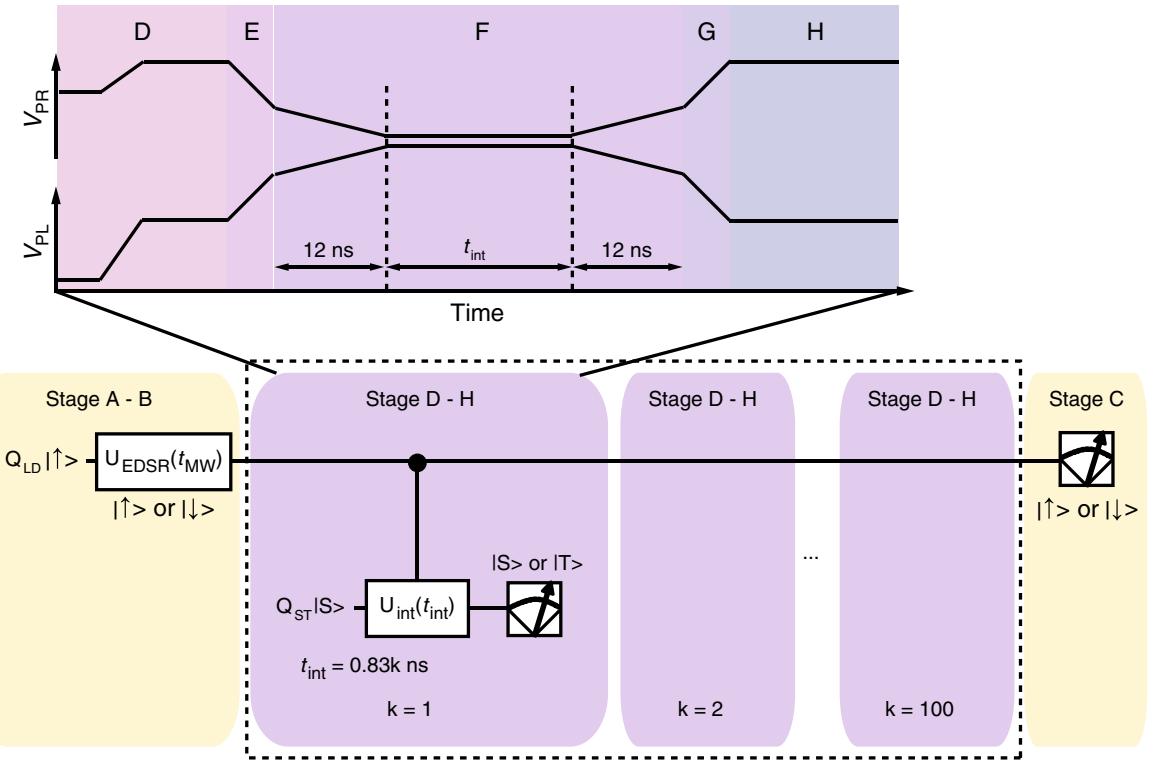

e

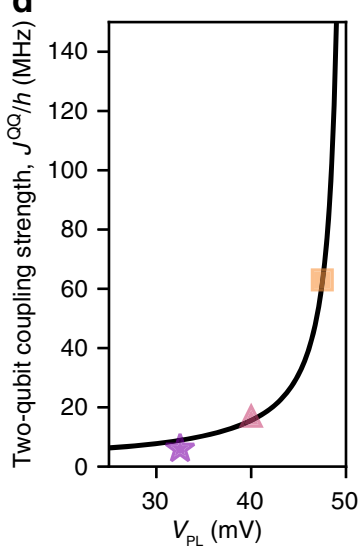

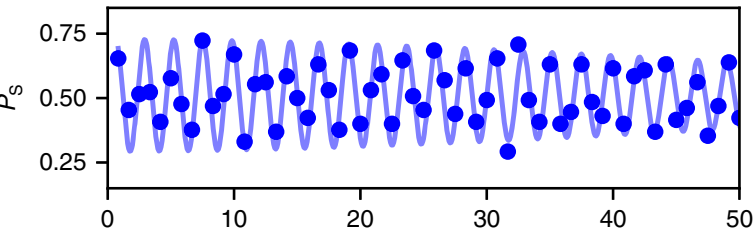

f

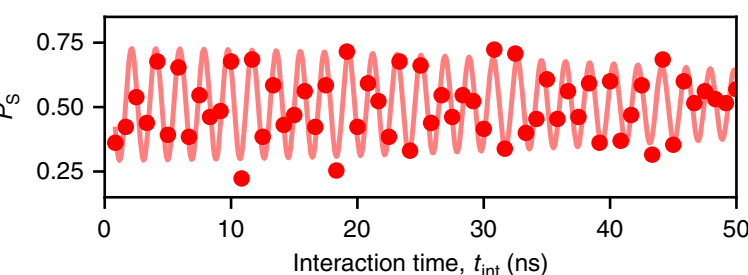

Fig. $2 \mathrm{ST}$ qubit frequency controlled by the LD qubit. a Energy diagram of the two-qubit states for $E_{\mathrm{Z}} \gg \Delta E_{\mathrm{Z}}^{\mathrm{ST}}, \Delta E_{\mathrm{Z}}^{\mathrm{QQ}} \gg J^{\mathrm{QQ}}\left(J^{S T}=0\right)$. The $\mathrm{ST}$ qubit frequency is equal to $\Delta E_{Z}^{S T}$ for $J Q Q=0$, and shifts by $\pm J Q Q / 2$ depending on the $\mathrm{Q}_{\mathrm{LD}}$ state for finite $J \mathrm{QQ}$. $\mathbf{b}$ The quantum circuit for demonstrating the phase control of $Q_{S T}$ depending on $Q_{L D}$. After preparing an arbitrary state of $Q_{L D}$ (stages A and B), we run modified stages from $D$ to $H$ (shown in the upper panel) 100 times with $t_{\text {int }}$ values ranging from 0.83 to 83 ns to observe the time evolution of $Q_{S T}$ without reinitializing or measuring $Q_{L D}$. Stages $A, B$ and C take $202 \mu \mathrm{s}$ in total and the part from D to $\mathrm{H}$ is $7 \mu$ s long. c FFT spectra of $f^{S T}$ with different interaction points shown by the white corresponding symbol in Fig. $1 \mathrm{~b}$ (traces offset for clarity). In addition to the frequency splitting due to JQQ, the center frequency of the two peaks shifts because $\Delta E_{\mathrm{Z}}^{\mathrm{ST}}$ is also dependent on the interaction point (Methods). $\mathbf{d}$ Interaction point dependence of the ST qubit frequency splitting, i.e. the two-qubit coupling strength JQQ/ $h$, fitted with the black model curve (see Supplementary Note 4 for the data extraction and fitting). e ST precession for the $Q_{L D}$ input state $|\uparrow\rangle$ fitted with the Gaussian-decaying oscillations with a decay time of $72 \mathrm{~ns}$. f ST precession for the $Q_{L D}$ input state $|\downarrow\rangle$ with a fitting curve. The decay time is $75 \mathrm{~ns}$. The total data acquisition time for $\mathbf{e}$ and $\mathbf{f}$ is $451 \mathrm{~ms}$

$\phi^{\mathrm{ST}}=2 \pi \Delta E_{\mathrm{Z}}^{\mathrm{ST}}\left(t_{\text {int }}+t_{\text {ramp }}\right) / h+\phi_{0}$, respectively. Here $t_{0}\left(\ll t_{\text {ramp }}\right)$ represents the effective time for switching on and off $J \mathrm{QQ}$ (Supplementary Note 5). A phase offset $\phi_{0}$ denotes the correction accounting for nonuniform $\Delta E_{\mathrm{Z}}^{\mathrm{ST}}$ during the ramp (Supplementary Note 5). Then the probability of finding the final state of $\mathrm{Q}_{\mathrm{ST}}$ in singlet is modeled as

$$
P_{\mathrm{S}, \text { model }}=a \cos \left(\phi_{\sigma_{z}^{\mathrm{LD}}}+\phi^{\mathrm{ST}}\right) \exp \left(-\left(t_{\text {int }} / T_{2}^{*}\right)\right)^{2}+b
$$

where $a, b$ and $T_{2}^{*}$ represent the values of amplitude, mean and the dephasing time of the ST precession, respectively. We use maximum likelihood estimation (MLE) combined with Bayesian estimation $^{29,30}$ to fit all variables in Eq. 2, that are $a, b, t_{0}, J^{\mathrm{QQ}}, T_{2}^{*}, \phi_{0}$, and $\Delta E_{\mathrm{Z}}^{\mathrm{ST}}$, from the data (Methods). This allows us to extract the $t_{\text {int }}$ dependence of $\phi_{\sigma^{\mathrm{LD}}}$ (Fig. 3a) (Methods) and consequently $\phi_{\mathrm{C}}=\phi_{|\downarrow\rangle}-\phi_{|\uparrow\rangle}$ (Fig. $3 \mathrm{~b}$ ). It evolves with $t_{\text {int }}$ in the frequency of $J^{\mathrm{QQ}} / \mathrm{h}=90 \mathrm{MHz}$, indicating that the CPHASE gate time can be as short as $h / 2 J^{\mathrm{QQ}}=5.5 \mathrm{~ns}$ (up to single-qubit phase). On the other hand, $T_{2}^{*}$ obtained in the MLE is $211 \mathrm{~ns}$, much longer than what is observed in Fig. $2 \mathrm{e}$, $\mathrm{f}$ because the shorter data acquisition time used here cuts off the lowfrequency component of the noise spectrum ${ }^{29}$. We note that this $T_{2}^{*}$ is that for the two-qubit gate while $J^{\mathrm{QQ}}$ is turned on ${ }^{8}$, and therefore it is likely to be dominated by charge noise rather than the nuclear field fluctuation (Supplementary Note 6). The ratio $2 J^{\mathrm{QQ}} T_{2}^{*} / h$ suggests that $38 \mathrm{CPHASE}$ operations would be possible within the two-qubit dephasing time. We anticipate that this ratio can be further enhanced by adopting approaches used to reduce 



Fig. 3 Controlled-phase evolution. a Interaction time $t_{\text {int }}$ dependence of $\phi_{\sigma_{2}^{\text {LD }}}$ controlled by $Q_{L D}$. The blue and red data are for $Q_{L D}=|\uparrow\rangle$ and $|\downarrow\rangle$, respectively. The solid curves are $\sin \left(\pi / \mathrm{QQ}\left(t_{\text {int }}+t_{0}\right) / h\right)$ (red) and $\sin$ $\left(-\pi J \mathrm{QQ}\left(t_{\text {int }}+t_{0}\right) / h\right)$ (blue) where the values of $J \mathrm{QQ}$ and $t_{0}$ are obtained in the MLE. The curves are consistent with the data as expected. $\mathbf{b}$ Controlledphase $\phi_{\mathrm{C}}=\phi_{|\downarrow\rangle}-\phi_{|\uparrow\rangle}$ extracted from Fig. 3a. Including the initial phase accumulated during gate voltage ramps at stage $\mathrm{F}, \phi_{\mathrm{C}}$ reaches $\pi$ first at $t_{\mathrm{int}}$ $=4.0 \mathrm{~ns}$ and increases by $\pi$ in every $5.5 \mathrm{~ns}$ afterwards

the sensitivity to charge noise in exchange gates such as symmetric operation ${ }^{31,32}$ and operation in an enhanced field gradient $^{33}$.

Finally we show that the CPHASE gate operates correctly for arbitrary $\mathrm{Q}_{\mathrm{LD}}$ input states. We implement the circuit shown in Fig. $4 \mathrm{a}$ in which $t_{\text {int }}$ is fixed to yield $\phi_{\mathrm{C}}=\pi$, while a coherent initial $\mathrm{Q}_{\mathrm{LD}}$ state with an arbitrary $\sigma_{z}^{\mathrm{LD}}$ is prepared by EDSR. We extract the averaged $\phi_{\sigma_{z}^{\mathrm{LD}}},\left\langle\phi_{\sigma_{z}^{\mathrm{LD}}}\right\rangle$ by Bayesian estimation ${ }^{29,30}$, which shows an oscillation as a function of $t_{\mathrm{MW}}$ in agreement with the Rabi oscillation measured independently by reading out $\mathrm{Q}_{\mathrm{LD}}$ at stage C as shown in Fig. 4b (see Methods for the estimation procedure and the origin of the low visibility, i.e., $\left.\max \left|\left\langle\phi_{\sigma^{\mathrm{LD}}}\right\rangle\right|<\pi / 2\right)$. These results clearly demonstrate the CPHASE gate functioning for an arbitrary $\mathrm{Q}_{\mathrm{LD}}$ input state.

\section{Discussion}

In summary, we have realized a fast quantum interface between a LD qubit and a ST qubit using a TQD. The CPHASE gate between these qubits is performed in $5.5 \mathrm{~ns}$, much faster than its dephasing time of $211 \mathrm{~ns}$ and those ratio ( 38) would be high enough to provide a high-fidelity CPHASE gate (Supplementary Note 8). Optimizing the magnet design to enhance the field gradient would allow even faster gate time beyond $\mathrm{GHz}$ with larger $J^{\mathrm{QQ}}$. At the same time, this technique is directly applicable to Si-based devices with much better single-qubit coherence ${ }^{5-9}$. Our results suggest that the performance of certain quantum computational tasks can be enhanced by adopting different kinds of qubits for different roles. For instance, LD qubits can be used for high-fidelity control and long memory and the ST qubit for fast initialization and readout. This combination is ideal for a


Fig. 4 Demonstration of the controlled-phase gate for arbitrary control qubit states. a The circuit for CPHASE gate demonstration. Here $t_{\text {int }}$ is fixed at $4.2 \mathrm{~ns}$ where $\phi_{\mathrm{C}} \approx \pi$ (Fig. 3b). $\mathbf{b} t_{\mathrm{MW}}$ dependence of the spin-down probability of $\mathrm{Q}_{\mathrm{LD}}, P_{\downarrow}$ (yellow) and the averaged $\phi_{\sigma_{2}^{\mathrm{L}}},\left\langle\phi_{\sigma_{\mathrm{L}}^{\mathrm{D}}}\right\rangle$ (purple) obtained by the circuit shown in Fig. 4a. $\left\langle\phi_{\sigma_{z}^{\mathrm{LD}}}\right\rangle\left(=-\pi\left\langle\sigma_{z}^{\mathrm{LD}}\right\rangle / 2\right)$ is expected to be proportional to $P_{\downarrow}$. We see $\left\langle\phi_{\sigma_{p}^{\mathrm{LD}}}\right\rangle$ oscillates depending on the input $\mathrm{Q}_{\mathrm{LD}}$ state. The oscillation visibility of $\left\langle\phi_{\sigma_{\mathrm{Z}}^{\mathrm{LD}}}\right\rangle$ is most probably limited by low preparation fidelity of the input $\mathrm{Q}_{L D}$ state as the visibility of the oscillation in $P_{\downarrow}$ is also low (see Methods)

example, the surface code quantum error correction where a data qubit must maintain the coherence while a syndrome qubit must be measured quickly ${ }^{34}$. Furthermore, the fast $\left(\sim 100 \mathrm{~ns}^{25}\right)$ ST qubit readout will allow the read out of a LD qubit in a quantumnon-demolition manner ${ }^{35}$ with a speed three orders of magnitude faster than a typical energy-selective tunneling measurement ${ }^{16,17}$. Viewed from the opposite side, we envisage coupling two ST qubits through an intermediate LD qubit, which would boost the two ST qubit gate speed by orders of magnitude compared to the demonstrated capacitive coupling scheme ${ }^{14}$. In addition, our results experimentally support the concept of the theoretical proposal of a fast two-qubit gate between two ST qubits based on direct exchange ${ }^{36}$ which shares the same working principle as our two-qubit gate. Our approach will further push the demonstrated scalability of spin qubits in quantum dot arrays beyond the conventional framework based on a unique spin-qubit encoding.

\section{Methods}

Device design. Our device was fabricated on a $\mathrm{GaAs} / \mathrm{Al}_{0.3} \mathrm{Ga}_{0.7} \mathrm{As}$ heterostructure wafer having a two-dimensional electron gas $100 \mathrm{~nm}$ below the surface, grown by molecular beam epitaxy on a semi-insulating (100) GaAs substrate. The electron density $n$ and mobility $\mu$ at a temperature of $4.2 \mathrm{~K}$ are $n=3.21 \times 10^{15} \mathrm{~m}^{-2}$ and $\mu=$ $86.5 \mathrm{~m}^{2} \mathrm{~V}^{-1} \mathrm{~s}^{-1}$ in the dark, respectively. We deposited $\mathrm{Ti} / \mathrm{Au}$ gate electrodes to define the TQD and the charge sensing single electron transistor. A piece of Co metal (micro-magnet, $\mathrm{MM}$ ) is directly placed on the surface of the wafer to provide a local magnetic field gradient in addition to the external magnetic field applied inplane (along $z$ ). The MM geometry is designed based on the numerical simulations of the local magnetic field ${ }^{23}$. The field property is essentially characterized by the two parameters ${ }^{23}: \mathrm{d} B_{x} / \mathrm{d} z$ at the position of each dot and the difference in $B_{z}$

between the neighboring dots, $\Delta B_{z}$ (see Fig. 1a for the definition of the $x$ and $z$ axes). $\mathrm{d} B_{x} / \mathrm{d} z$ determines the spin rotation speed by EDSR and is as large as $\sim 1 \mathrm{mT}$ $\mathrm{nm}^{-1}$ at the left dot (Supplementary Fig. 5a) allowing fast control of $\mathrm{Q}_{\mathrm{LD}}\left(f_{\mathrm{Rabi}}>\right.$ $10 \mathrm{MHz})^{20,23}$. At the same time $\Delta B_{z}$ between the left and center dots, $\Delta B_{z}^{\mathrm{LC}}$, is designed to be $\sim 60 \mathrm{mT}$ (Supplementary Fig. 5b) to guarantee the selective EDSR 
control of $\mathrm{Q}_{\mathrm{LD}}$ without rotating the spin in the center $\operatorname{dot}^{20,23}$. Furthermore, $\Delta B_{z}$ between the center and right dots, $\Delta B_{z}^{\mathrm{CR}}$, is designed to be $\sim 40 \mathrm{mT}$ (Supplementary Fig. 5b) to make the eigenstates of $\mathrm{Q}_{S \mathrm{~T}}|\uparrow \downarrow\rangle$ and $|\downarrow \uparrow\rangle$ rather than $|S\rangle$ and $|\mathrm{T}\rangle$ by satisfying $\Delta E_{\mathrm{Z}}^{\mathrm{ST}} \gg J^{\mathrm{ST}}$. Note that $\Delta E_{\mathrm{Z}}^{\mathrm{ST}}=|g| \mu_{\mathrm{B}} \Delta B_{z}^{\mathrm{CR}}$ where $g \sim-0.4$ and $\mu_{\mathrm{B}}$ are the electron $g$-factor and Bohr magneton, respectively. From the design we expect a large variation of $\Delta B_{z}^{\mathrm{CR}}$ when the electron in the center dot is displaced by the electric field. Indeed, we observe a strong influence of the gate voltages on $\Delta B_{z}^{\mathrm{CR}}$, which reaches $\sim 100 \mathrm{mT}\left(\Delta E_{\mathrm{Z}}^{\mathrm{ST}} / h \sim 500 \mathrm{MHz}\right)$ in the configuration chosen for the two-qubit gate experiment.

Estimation of the ST precession parameters. Here we describe the estimation of the ST precession parameters in Eq. 2 under the influence of a fluctuating singlequbit phase of $\mathrm{Q}_{\mathrm{ST}}$. Out of the parameters involved, $\phi_{\sigma^{\mathrm{LD}}}$ is the only parameter assumed to be $\mathrm{Q}_{\mathrm{LD}}$ state-dependent, and the rest is classified into two types. One is the pulse-cycle-independent parameters, $a, b, J^{\mathrm{QQ}}, T_{2}^{*}$ and $t_{0}$ which is constant during the experiment, and the other is the pulse-cycle-dependent parameters, $\sigma_{z}^{\mathrm{LD}}, \Delta E_{\mathrm{Z}}^{\mathrm{ST}}$ and $\phi_{0}$, which can change cycle by cycle. Each pulse cycle consists of pulse stages from $\mathrm{A}$ to $\mathrm{C}$ as shown in Fig. $2 \mathrm{~b}$. We run the pulse cycle consecutively with a MW frequency fixed at $17.26 \mathrm{GHz}$ and collect the data while $\mathrm{Q}_{\mathrm{LD}}$ drifts between on-resonances and off-resonances with the MW burst due to the nuclear field fluctuation. To decrease the uncertainty of the estimated parameters, we choose the cycles during which the spin flip of $\mathrm{Q}_{\mathrm{LD}}$ is unlikely in the following manner. The cycles throughout which $\mathrm{Q}_{\mathrm{LD}}$ is likely to be $|\downarrow\rangle$ are post-selected by the condition that $\mathrm{Q}_{\mathrm{LD}}$ is on-resonance (i.e., Rabi oscillation of $\mathrm{Q}_{\mathrm{LD}}$ is observed in ensemble-averaged data from nearby cycles) and the final state of $\mathrm{Q}_{\mathrm{LD}}$ is measured to be $|\downarrow\rangle$ at pulse stage $C$. Similarly, the cycles for $Q_{L D}=|\uparrow\rangle$ are post-selected by the condition that $\mathrm{Q}_{\mathrm{LD}}$ is off-resonance and the final state of $\mathrm{Q}_{\mathrm{LD}}$ is measured to be $|\uparrow\rangle$. The data structure and the index definitions for MLE are summarized in Supplementary Table $1 . k$ is the index of the interaction time such that $t_{\text {int }}=0.83 \times k$ ns with $k$ ranging from 1 to $100 . m$ is the pulse-cycle index ranging from 1 (2001) to $2000(4000)$ for $\mathrm{Q}_{\mathrm{LD}}$ prepared in $|\uparrow\rangle(|\downarrow\rangle)$. The estimation procedure is the following. From all the readout results of $\mathrm{Q}_{\text {ST }}($ stage $\mathrm{H}$ ) obtained in the cycles, we first estimate the five pulse-cycle-independent parameters by MLE. Note that $J^{\mathrm{QQ}}$ may have a small pulse-cycle-dependent component due to charge noise but this effect is captured as additional fluctuation in $\Delta E_{\mathrm{Z}}^{\mathrm{ST}}$ and $\phi_{0}$ in our model. We apply MLE to $100 \times 4000$ readout results of $\mathrm{Q}_{\mathrm{ST}}, r_{m}^{k}=1(0)$ for $\mathrm{Q}_{\mathrm{ST}}=|\mathrm{S}\rangle(|\mathrm{T}\rangle)$. To this end, we first introduce the likelihood $P_{m}$ defined in the eight dimensional parameter space as

$$
P_{m}\left(a, b, t_{0}, J^{\mathrm{QQ}}, T_{2}^{*}, \sigma_{z}^{\mathrm{LD}}, \phi_{0}, \Delta E_{\mathrm{Z}}^{\mathrm{ST}}\right)=\prod_{k=1}^{100}\left(r_{m}^{k} P_{\mathrm{S}, \text { model }}+\left(1-r_{m}^{k}\right)\left(1-P_{\mathrm{S}, \text { model }}\right)\right)
$$

where $P_{\mathrm{S} \text {,model }}$ is defined in Eq. (2). We calculate $P_{m}$ on a discretized space within a chosen parameter range (Supplementary Table 2) using a single cycle data. Then we obtain $P_{m}$ for the target five parameters as a marginal distribution by tracing out the pulse-cycle-dependent parameters,

$$
P_{\mathrm{m}}\left(a, b, t_{0}, J^{\mathrm{QQ}}, T_{2}^{*}\right)=\sum_{\sigma_{z}^{\mathrm{LD}}} \sum_{\phi_{0}} \sum_{\Delta E_{\mathrm{Z}}^{\mathrm{ST}}} P_{m}\left(a, b, t_{0}, J^{\mathrm{QQ}}, T_{2}^{*}, \sigma_{z}^{\mathrm{LD}}, \phi_{0}, \Delta E_{\mathrm{Z}}^{\mathrm{ST}}\right) .
$$

Repeating this process for all pulse cycles, we obtain the likelihood $P$ as

$$
P\left(a, b, t_{0}, J^{\mathrm{QQ}}, T_{2}^{*}\right)=\prod_{m} P_{m}\left(a, b, t_{0}, J^{\mathrm{QQ}}, T_{2}^{*}\right) .
$$

We choose the maximum of $P$ as the estimator for $a, b, t_{0}, J^{\mathrm{QQ}}$ and $T_{2}^{*}$, obtaining $a=0.218 \pm 0.005, b=0.511 \pm 0.003, t_{0}=1.53 \pm 0.17 \mathrm{~ns}, J^{Q Q} / h=90.2 \pm$ $0.3 \mathrm{MHz}, T_{2}^{*}=211 \pm 37 \mathrm{~ns}$.

Once these values are fixed, we estimate the pulse-cycle-dependent parameters, $\sigma_{z}^{\mathrm{LD}}, \phi_{0}$ and $\Delta E_{\mathrm{Z}}^{\mathrm{ST}}$, for each cycle $m$. Note that $\sigma_{z}^{\mathrm{LD}}$ could be prepared deterministically if the state preparation of $\mathrm{Q}_{\mathrm{LD}}$ were ideal, but here we treat it as one of the parameters to be estimated because of a finite error in the $\mathrm{Q}_{\mathrm{LD}}$ state preparation. We again evaluate the likelihood $P_{m}\left(\sigma_{z}^{\mathrm{LD}}, \phi_{0}, \Delta E_{\mathrm{Z}}^{\mathrm{ST}}\right)$ defined in a discretized three dimensional space of its parameters using Eq. 3 and find their values that maximize the likelihood.

Based on the values of $a, b, T_{2}^{*}$ and $\phi^{\mathrm{ST}}$ determined above, we can directly estimate $\phi_{\sigma_{2}^{\mathrm{LD}}}$ controlled by $\mathrm{Q}_{\mathrm{LD}}$ for each $t_{\text {int }}$ without presumptions on the value of $J^{\mathrm{QQ}}$. To this end, we search for the parameter $\phi_{\sigma_{2}^{\mathrm{D}}}$ that maximizes the likelihood

$$
P^{k}\left(\phi_{\sigma_{z}^{\text {LD }}}\right)=\prod_{m}\left(r_{m}^{k} P_{\mathrm{S}, \text { model }}+\left(1-r_{m}^{k}\right)\left(1-P_{\mathrm{S}, \text { model }}\right)\right) .
$$

The obtained estimators for $\phi_{|\downarrow\rangle}$ and $\phi_{|\uparrow\rangle}$ are consistent with the expected values $\pm \pi J^{\mathrm{QQ}}\left(t_{\text {int }}+t_{0}\right) / h$ calculated from $J \mathrm{QQ} / h$ and $t_{0}$ found above (see Fig. $3 \mathrm{a}$ ).

The ensemble-averaged phase $\left\langle\phi_{\sigma_{z}^{\mathrm{LD}}}\right\rangle$ is obtained based on a similar estimation protocol. Here we estimate $\phi_{\sigma_{\mathrm{LD}}}$ for each $m$ with fixed $k=5\left(t_{\text {int }}=4.2 \mathrm{~ns}\right)$ to yield
$\phi_{\mathrm{C}} \approx \pi$ from the likelihood $P_{m}^{k=5}=r_{m}^{k=5} P_{\mathrm{S}, \text { model }}+\left(1-r_{m}^{k=5}\right)\left(1-P_{\mathrm{S}, \text { model }}\right)$ and then take the average of the estimated values for 800 pulse cycles. The oscillation visibility of $\left\langle\phi_{\sigma_{z}^{\text {एD }}}\right\rangle$ in Fig. $4 \mathrm{~b}$ is limited by three factors, low preparation fidelity of the input $\mathrm{Q}_{\mathrm{LD}}$ state, estimation error of $\phi_{\sigma_{2}^{\mathrm{LD}}}$ and CPHASE gate error. The first contribution is likely to be dominant as the visibility of the oscillation in $\mathrm{P}_{\downarrow}$ is correspondingly low. Note that the effect of those errors is not visible in Fig. 3 because the most likely values of $\phi_{\sigma_{2}^{\mathrm{LD}}}$ are plotted.

\section{Data availability}

The data that support the findings of this study are available from the corresponding authors upon reasonable request.

Received: 20 August 2018 Accepted: 8 November 2018 Published online: 29 November 2018

\section{References}

1. Nielsen, M. A. \& Chuang, I. L. Quantum Computation and Quantum Information. Cambridge Series on Information and the Natural Sciences (Cambridge University Press, Cambridge, UK, 2000).

2. Martinis, J. M. Qubit metrology for building a fault-tolerant quantum computer. npj Quantum Inf. 1, 15005 (2015).

3. Loss, D. \& DiVincenzo, D. P. Quantum Computation with Quantum Dots. Phys. Rev. A 57, 120-126 (1998).

4. Koppens, F. H. L. et al. Driven coherent oscillations of a single electron spin in a quantum dot. Nature 442, 766-771 (2006).

5. Yoneda, J. et al. A quantum-dot spin qubit with coherence limited by charge noise and fidelity higher than 99.9\%. Nat. Nanotechnol. 13, 102-106 (2018).

6. Zajac, D. M. et al. Resonantly driven CNOT gate for electron spins. Science 359, 439-442 (2017).

7. Watson, T. F. et al. A programmable two-qubit quantum processor in silicon Nature 555, 633-637 (2018)

8. Veldhorst, M. et al. A two-qubit logic gate in silicon. Nature 526, 410-414 (2015).

9. Veldhorst, M. et al. An addressable quantum dot qubit with fault-tolerant control-fidelity. Nat. Nanotechnol. 9, 981-985 (2014).

10. Taylor, J. M. et al. Fault-tolerant architecture for quantum computation using electrically controlled semiconductor spins. Nat. Phys. 1, 177-183 (2005).

11. Petta, J. R. et al. Coherent manipulation of coupled electron spins in semiconductor quantum dots. Science 309, 2180-2184 (2005).

12. Barthel, C. et al. Rapid single-shot measurement of a singlet-triplet qubit. Phys. Rev. Lett. 103, 160503 (2009).

13. Nakajima, T. et al. Robust single-shot spin measurement with $99.5 \%$ fidelity in a quantum dot array. Phys. Rev. Lett. 119, 017701 (2017).

14. Shulman, M. D. et al. Demonstration of entanglement of electrostatically coupled singlet-triplet qubits. Science 336, 202-205 (2012).

15. Medford, J. et al. Self-consistent measurement and state tomography of an exchange-only spin qubit. Nat. Nanotechnol. 8, 654-659 (2013).

16. Elzerman, J. M. et al. Single-shot read-out of an individual electron spin in a quantum dot. Nature 430, 431-435 (2004).

17. Baart, T. et al. Single-spin CCD. Nat. Nanotechnol. 11, 330-334 (2016).

18. Mehl, S. \& DiVincenzo, D. P. Simple operation sequences to couple and interchange quantum information between spin qubits of different kinds. Phys. Rev. B 92, 115448 (2015).

19. Trifunovic, L. et al. D. Long-distance spin-spin coupling via floating gates. Phys. Rev. X 2, 011006 (2012).

20. Yoneda, J. et al. Fast electrical control of single electron spins in quantum dots with vanishing influence from nuclear spins. Phys. Rev. Lett. 113, 267601 (2014).

21. Pioro-Ladrière, M. et al. Electrically driven single-electron spin resonance in a slanting Zeeman field. Nat. Phys. 4, 776-779 (2008).

22. Tokura, Y., van der Wiel, W. G., Obata, T. \& Tarucha, S. Coherent Single Electron Spin Control in a Slanting Zeeman Field. Phys. Rev. Lett. 96, 047202 (2006).

23. Yoneda, J. et al. Robust micromagnet design for fast electrical manipulations of single spins in quantum dots. Appl. Phys. Express 8, 084401 (2015).

24. Reilly, D. J., Marcus, C. M., Hanson, M. P. \& Gossard, A. C. Fast single-charge sensing with a rf quantum point contact. Appl. Phys. Lett. 91, 162101 (2007).

25. Barthel, C. et al. Fast sensing of double-dot charge arrangement and spin state with a radio-frequency sensor quantum dot. Phys. Rev. B 81, 161308(R) (2010).

26. Noiri, A. et al. Coherent electron-spin-resonance manipulation of three individual spins in a triple quantum dot. Appl. Phys. Lett. 108, 153101 (2016).

27. Barthel, C. et al. Relaxation and readout visibility of a singlet-triplet qubit in an Overhauser field gradient. Phys. Rev. B 85, 035306 (2012). 
28. Nakajima, T. et al. Coherent transfer of electron spin correlations assisted by dephasing noise. Nat. Commun. 9, 2133 (2018).

29. Delbecq, R. M. et al. Quantum dephasing in a gated GaAs triple quantum dot due to nonergodic noise. Phys. Rev. Lett. 116, 046802 (2016).

30. Shulman, M. D. et al. Suppressing qubit dephasing using real-time Hamiltonian estimation. Nat. Comm. 5, 5156 (2014).

31. Martins, F. et al. Noise suppression using symmetric exchange gates in spin qubits. Phys. Rev. Lett. 116, 116801 (2016).

32. Reed, M. D. et al. Reduced sensitivity to charge noise in semiconductor spin qubits via symmetric operation. Phys. Rev. Lett. 116, 110402 (2016).

33. Nichol, J. M. et al. High-fidelity entangling gate for double-quantum-dot spin qubits. npj Quantum Inf. 3, 3 (2017).

34. Fowler, A. G., Mariantoni, M., Martinis, J. M. \& Cleland, A. N. Surface codes: towards practical large-scale quantum computation. Phys. Rev. A 86, 032324 (2012).

35. Braginsky, V. B. \& Khalili, F. Ya Quantum nondemolition measurements: the route from toys to tools. Rev. Mod. Phys. 68, 1-11 (1996).

36. Wardrop, M. P. \& Doherty, A. C. Exchange-based two-qubit gate for singlettriplet qubits. Phys. Rev. B 90, 045418 (2014).

\section{Acknowledgements}

Part of this work is financially supported by the ImPACT Program of Council for Science, Technology and Innovation (Cabinet Office, Government of Japan), the Grantin-Aid for Scientific Research (No. 26220710), CREST (JPMJCR15N2, JPMJCR1675), JST, Incentive Research Project from RIKEN. A.N. acknowledges support from Advanced Leading Graduate Course for Photon Science (ALPS). T.N. acknowledges financial support from JSPS KAKENHI Grant Number 18H01819. T.O. acknowledges financial support from Grants-in-Aid for Scientific Research (No. 16H00817, 17H05187), PRESTO (JPMJPR16N3), JST, The Telecommunications Advancement Foundation Research Grant, Futaba Electronics Memorial Foundation Research Grant, Hitachi Global Foundation Kurata Grant, The Okawa Foundation for Information and Telecommunications Research Grant, The Nakajima Foundation Research Grant, Japan Prize Foundation Research Grant, Iketani Science and Technology Foundation Research Grant,Yamaguchi Foundation Research Grant, Kato Foundation for Promotion of Science Research Grant. A.D.W. and A.L. acknowledge gratefully support of DFG-TRR160, BMBF - Q.Link.X 16KIS0867, and the DFH/UFA CDFA-05-06.

\section{Author contributions}

A.N. and J.Y. conceived the experiment. A.N. and T.N. performed the measurement with the assistance of K.K., Y.K., M.R.D., T.O., K.T., S.A., and G.A. A.N. and T.N. conducted data analysis with the inputs from J.Y., P.S., and D.L. A.N. and T.N. fabricated the device on the heterostructure grown by A.L. and A.D.W. A.N. and T.N. wrote the manuscript with inputs from other authors. All authors discussed the results. The project was supervised by S.T.

\section{Additional information}

Supplementary Information accompanies this paper at https://doi.org/10.1038/s41467018-07522-1.

Competing interests: The authors declare no competing interests.

Reprints and permission information is available online at http://npg.nature.com/ reprintsandpermissions/

Publisher's note: Springer Nature remains neutral with regard to jurisdictional claims in published maps and institutional affiliations.

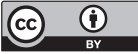

Open Access This article is licensed under a Creative Commons Attribution 4.0 International License, which permits use, sharing, adaptation, distribution and reproduction in any medium or format, as long as you give appropriate credit to the original author(s) and the source, provide a link to the Creative Commons license, and indicate if changes were made. The images or other third party material in this article are included in the article's Creative Commons license, unless indicated otherwise in a credit line to the material. If material is not included in the article's Creative Commons license and your intended use is not permitted by statutory regulation or exceeds the permitted use, you will need to obtain permission directly from the copyright holder. To view a copy of this license, visit http://creativecommons.org/ licenses/by/4.0/.

(C) The Author(s) 2018 